\title{
Marine benthic flora of the Dampier Archipelago, Western Australia
}

\author{
John M. Huisman \\ School of Biological Sciences and Biotechnology, Murdoch University, Murdoch, \\ Western Australia 6150, Australia \\ email: J.Huisman@murdoch.edu.au
}

\begin{abstract}
There is a lack of information regarding the marine benthic flora of northwest Western Australia and a comprehensive flora for the region has never been produced. A checklist of the marine benthic flora of the Dampier Archipelago collected during the Western Australian Museum expeditions of $1998 / 1999$ is presented. This list, however, must be viewed as preliminary, as many of the specimens collected are yet to be identified.
\end{abstract}

\section{INTRODUCTION}

The marine benthic flora includes photosynthetic organisms from a diverse range of taxonomic groups, including green, brown, and red algae (Chlorophyta, Phaeophyceae and Rhodophyta, respectively), angiosperms (Magnoliophyta) and bacteria (Cyanophyta or Cyanobacteria). Several recent publications have highlighted the lack of information regarding the marine benthic flora of northwest Western Australia (e.g. Huisman et al., 1998). A compendium of records of marine algae from northern Australia (Lewis, 1984; 1985; 1987) included some 800 species, of which only nine were attributed to the Western Australian coast north of (and including) Northwest Cape. Several recent publications have increased the number of species recorded for the region (e.g. Phillips et al., 1993; King and Puttock, 1994; Huisman, 1996; Phillips and Huisman, 1997; Phillips, 2000; Huisman, 2001; 2002) and many tropical species from the northwest were illustrated in the recent Marine Plants of Australia (Huisman, 2000). However, a comprehensive flora for the region has yet to be produced. This report provides a checklist of the marine benthic flora of the Dampier Archipelago collected during the Western Australian Museum expeditions of $1998 / 1999$. It should be treated as a companion piece to Huisman and Borowitzka
(2003), which provides a more detailed list of species, including many not recorded during the museum expeditions. Both this list and that of Huisman and Borowitzka (2003) must be viewed as preliminary, however, as many of the specimens collected are yet to be identified.

\section{MATERIALS AND METHODS}

Specimens were collected by SCUBA, snorkel, or from the intertidal and preserved in a $5-10 \%$ solution of formalin/seawater. Refer to the Station Lists section of this volume for the diving (DA1/98, DA3/99) and dredging (DA2/99) expedition station data. Slide material was stained in a mixture of $1 \mathrm{~g}$ aniline blue powder, $70 \mathrm{~mL}$ Karoß, $30 \mathrm{~mL}$ distilled water and $5 \mathrm{~mL}$ acetic acid. All specimens are presently housed in the Herbarium of the School of Biological Sciences and Biotechnology, Murdoch University (MURU). Descriptions and locations of sites are given elsewhere in this volume (see Station Lists). The species are arranged into Divisions (Chlorophyta $=$ green algae, Heterokontophyta: Phaeophyceae $=$ brown algae, Rhodophyta $=$ red algae, Cyanophyta = cyanobacteria = blue-green algae, Magnoliophyta = seagrasses), which are subdivided into families, generally arranged according to Silva et al., 1996. 


\section{CHECKLIST}

A Checklist of the Marine Benthic Flora of the Dampier Archipelago, Western Australia Site Number*

Numbers refer to diving expedition (DA1/98 and DA3/99) sites; dredging expedition site numbers are prefixed by DA2/99

Species

Site Number*

\section{CHLOROPHYTA}

\section{CHAETOPHORALES}

Ulvellaceae

Stromatella monostromatica (Dangeard) Kornmann 48

and Sahling, 1985

Ulvella lens P. Crouan and H. Crouan, 1859

48

45

\section{ULVALES}

\section{Ulvaceae}

Enteromorpha flexuosa (Wulfen) J. Agardh, 1883

Enteromorpha flexuosa (Wulfen) J. Agardh subsp. paradoxa (C. Agardh) Bliding, 1963

\section{CLADOPHORALES}

Anadyomenaceae

Anadyomene brownii (J.E. Gray) J. Agardh, 1887

\section{Siphonocladaceae}

Boergesenia forbesii (Harv.) Feldmann, 1938

Boodlea composita (Harv.) Brand, 1904

Cladophoropsissp.

Dictyosphaeria cavernosa (Forssk.) Børgesen, 1932

Dictyosphaeria versluysii Weber-van Bosse, 1905

Siphonocladus tropicus (P. Crouan and H. Crouan)

J. Agardh, 1887

\section{Valoniaceae}

Valonia aegagropila C. Agardh, 1823

Valoniopsis pachynema (Martens) Børgesen, 1934

Ventricaria ventricosa (J. Agardh) Olsen and J. West, 1988

BRYOPSIDALES

Bryopsidaceae

Pseudobryopsis hainanensis Tseng, 1936

\section{Caulerpaceae}

Caulerpa brachypus Harv., 1860

Caulerpa constricta Price, Huisman and Borowitzka, 1998

Caulerpa corynephora Montagne, 1842

Caulerpa cupressoides (Vahl) C. Agardh, 1817

Caulerpa lentillifera J. Agardh, 1837

Caulerpa racemosa (Forssk.) J. Agardh, 1873

Caulerpa racemosa (Forssk.) J. Agardh var. lamourouxii (Turner) Weber-van Bosse, 1898

Caulerpa racemosa (Forssk.) J. Agardh var. laetevirens (Montagne) Weber-van Bosse, 1898

Caulerpa racemosa (Forssk.) J. Agardh var. peltata

(J.V. Lamour.) Eubank, 1944
59,62

54
$03,17,25,28$

$03,31,58$

$45,47,48,70$

$06,17,31,38,45,51,54,70$

37

48,52

$09,37,42,61,70$

$25,28,54,61,70$

$06,23,31$

$10,54,56,61$

54,70

$16,17,25,61$ 
Caulerpa serrulata (Forssk.) J. Agardh, 1837

Caulerpa sertularioides (S.G. Gmelin) Howe, 1905

Caulerpa taxifolia (Vahl) C. Agardh, 1817

Caulerpa verticillata J. Agardh, 1847

Caulerpa webbiana Montagne, 1837

\section{Codiaceae}

Codium arabicum Kütz., 1856

Codium dwarkense Børgesen, 1847

Halimedaceae

Halimeda cuneata Hering in Krauss, 1846

Halimeda cylindracea Decne., 1842

Halimeda discoidea Decne., 1842

Halimeda macroloba Decne., 1841

Halimeda velasquezii W.R. Taylor, 1962

\section{Udoteaceae}

Avrainvillea obscura (C. Agardh) J. Agardh, 1887

Penicillus nodulosus Blainville, 1834

Udotea argentea Zanardini, 1858

Udotea flabellum (Ellis and Sol.) Howe, 1904

Udotea glaucescens Harv. ex J. Agardh, 1887

Udotea orientalis A. Gepp and E. Gepp, 1911

DASYCLADALES

\section{Dasycladaceae}

Bornetella oligospora Solms-Laubach, 1892

Bornetella sphaerica (Zanardini) Solms-Laubach, 1892

Neomeris vanbosseae Howe, 1909

Polyphysaceae

Acetabularia calyculus J.V. Lamour., 1824

\section{HETEROKONTOPHYTA}

Phaeophyceae

\section{ECTOCARPALES}

Ectocarpaceae

Feldmannia indica (Sond.) Womersley and Bailey, 1970

Hincksia mitchelliae (Harv.) Silva, 1987

SPHACELARIALES

Sphacelariaceae

Sphacelaria rigidula Kütz., 1843

Sphacelaria tribuloides Meneghini, 1840

\section{CUTLERIALES}

Cutleriaceae

Cutleria kraftii Huisman, 2000
47,62

37

$07,25,32,37,42,61,66$

$06,09,16,17,23,25,31,37,38,42,54,61,62$

06,56

$03,18,47$

$40,47,53,58$

51

51

$37,47,68$

$03,09,14,19,25,28,37,41,42,48,51,58,61$, $64,68, \mathrm{DA} 2 / 99 / 95$

56

$14,17,54,58$

$09,25,53$

51,59

$51,54,59$

$09,37,38,45,48,51,56,58,56,68,91$

09,14

41

56,65

$06,19,25,37,47,48,61$

06,25

$06,25,36,37$

56

59

48,62 


\section{DICTYOTALES}

\section{Dictyotaceae}

Dictyopteris australis (Sond.) Askenasy, in Engler, 1888

Dictyopteris delicatula J.V. Lamour., 1809

Dictyopteris woodwardia (R. Brown ex Turner) C. Agardh, 1817

Dictyota cervicornis Kütz., 1859

Dictyota ciliolata Sonder ex Kütz., 1859

Dictyota friabilis Setchell, 1926

Lobophora variegata (J.V. Lamour.) Womersley ex Oliviera, 1977

Padina australis Hauck, 1887

Padina elegans Koh ex Womersley, 1987

Padina tenuis Bory, 1827

Spatoglossum macrodontum J. Agardh, 1882

Stoechospermum polypodioides (J.V. Lamour) J. Agardh, 1848

Stypopodium flabelliforme Weber-van Bosse, 1913

\section{SCYTOSIPHONALES}

\section{Chnoosporaceae}

Chnoospora implexa J. Agardh, 1848

\section{Scytosiphonaceae}

Colpomenia sinuosa (Mertens ex Roth) Derbès and Solier

in Castagne, 1851

Hydroclathrus clathratus (C. Agardh) Howe, 1920

Rosenvingea nhatrangensis Dawson, 1954

\section{FUCALES}

Cystoseiraceae

Cystoseira trinodis (Forssk.) C. Agardh, 1820

Hormophysa cuneiformis (Gmelin) Silva, 1897

\section{Sargassaceae}

Sargassum decurrens (R. Brown ex Turner) C. Agardh, 1820

Sargassum oligocystum Montagne, 1845

Sargassum polycystum C. Agardh, 1824

Turbinaria gracilis Sonder, 1845

Turbinaria ornata (Turner) J. Agardh, 1848
$01,06,07,09,14,23,30,31,37,54,56,57,61$, $\mathrm{DA} 2 / 99 / 29$, DA2/99/42, DA2/99/49,

DA2/99/57, DA2/99/88, DA2/99/90 18

37,47

$07,25,37,38,45,61$

$10,25,37$

$16,27,67$

$07,09,13,16,23,29,36,37,40,41,42,45,47$, $57,58,69$

$01,07,10,16,28,48,54,61$

70

$03,13,16,17,31,34,40,66,70$

$11,16,37, \mathrm{DA} 2 / 99 / 47, \mathrm{DA} 2 / 99 / 88$

09

$23,31,40$

14

$01,03.4,25,37,38,45,51,57$

$07,17,34,45,47,54,62$

23

$01,09,14,23,31,37,45$

14

$09,16,37$

$09,16,25,30,37,45$

38,45

$01,45,64$

$03,38,42,58$

\section{RHODOPHYTA}

\section{GONIOTRICHALES}

Goniotrichaceae

Stylonema alsidii (Zanardini) Drew, 1956

\section{ERYTHROPELTIDALES}

Erythrotrichiaceae

Erythrotrichia carnea (Dillwyn) J. Agardh, 1883

\section{ACROCHAETIALES}

\section{Acrochaetiaceae}

Acrochaetium microscopicum (Nägeli ex Kütz.) Nägeli, 1858

Acrochaetium savianum (Meneghini) Nägeli, 1862 


\section{NEMALIALES}

\section{Galaxauraceae}

Galaxaura marginata (J. Ellis and Sol.) J.V. Lamour., 1816

Galaxaura obtusata (J. Ellis and Sol.) J.V. Lamour., 1816

Galaxaura rugosa (J. Ellis and Sol.) J.V. Lamour., 1816

Scinaia tsinglanensis Tseng, 1941

Tricleocarpa cylindrica (J. Ellis and Sol.) Huisman and Borowitzka, 1990

\section{Liagoraceae}

Ganonema farinosum (J.V. Lamour.) K.C. Fan and Yung C. Wang, 1974

Ganonema pinnatum (Harv.) Huisman, 2002

Ganonema borowitzkae Huisman, 2002

Liagora ceranoides J.V. Lamour., 1816

Liagora divaricata Tseng, 1941

Liagora valida Harv., 1853

Patenocarpus paraphysiferus Yoshizaki, 1987

Yamadaella caenomyce (Decne.) Abbott, 1970

Trichogloea requienii (Mont.) Kütz., 1847

\section{GELIDIALES}

Gelidiaceae

Pterocladiella caerulescens (Kützing) Santelices and Hommersand, 1997

\section{Gelidiellaceae}

Gelidiella acerosa (Forssk.) Feldmann and Hamel, 1934

GRACILARIALES

\section{Gracilariaceae}

Gracilaria blodgettii Harvey, 1853

Gracilaria canaliculata Sonder, 1871

Gracilaria salicornia (C. Agardh) Dawson, 1954

Gracilaria urvillei (Mont.) Abbott, 1991

BONNEMAISONIALES

Bonnemaisoniaceae

Asparagopsis taxiformis (Delile) Trevisan, 1845

CORALLINALES

\section{Corallinaceae}

Amphiroa foliacea J.V. Lamour., 1824

Amphiroa fragilissima (L.) J.V. Lamour., 1816

Haliptilon roseum (Lamarck) Garbary and Johansen, 1982

Jania adhaerens J.V. Lamour., 1816
$22,25,32$

22

14

69

\author{
$04,13,27,32,44,53,57$ \\ 27,56 \\ $01,03,09,16,31,32,37,45$ \\ 56 \\ $06,09,32$
}

$01,21,37$

47
38
14
68
18
45,47
03,45
13,16

39

68

$03,46,48$

$02,07,17,23,62$

06,48

\section{CERAMIALES}

\section{Ceramiaceae}

Aglaothamnion cordatum (Børgesen) Feldmann-Mazoyer, 1941 Anotrichium tenue (C. Agardh) Nägeli, 1862

Balliella subcorticata (Itono) Itono and Tanaka, 1973

Centroceras clavulatum (C. Agardh) Montagne, 1846

Ceramium affine Setchell and Gardner, 1930

Ceramium fimbriatum Setchell and Gardner, 1924

55

37

39,46

59

34,47

59 
Species

Ceramium flaccidum (Harv. ex Kütz.) Ardissone, 1871

Ceramium isogonum Harv., 1855

Ceramium macilentum J. Agardh, 1894

Ceramium borneense Weber-van Bosse, 1923

Crouania attenuata (C. Agardh) J. Agardh, 1842

Griffithsia metcalfii Tseng, 1942

Monosporus indicus Børgesen, 1931

Seirospora orientalis Kraft 1988

Spyridia filamentosa (Wulfen) Harv., 1833

Wrangelia sp.

\section{Dasyaceae}

Dasya sp.

Heterosiphonia crassipes (Harv.) Falkenb., 1901

\section{Delesseriaceae}

Calloglossa leprieurii (Montagne) Martens, 1869

Cottoniella filamentosa (Howe) Børgesen, 1920

Cottoniella amamiensis Itono, 1972

Hypoglossum caloglossoides Wynne and Kraft, 1985

Martensia fragilis Harv., 1854

Platysiphonia delicata (Clemente) Cremades, 1990

Taenioma perpusillum (J. Agardh) J. Agardh, 1863

\section{Rhodomelaceae}

Acanthophora spicifera (Vahl) Børgesen, 1910

Bostrychia radicans (Montagne) Montagne, 1842

Chondria armata (Kütz.) Okamura, 1907-1909

Chondria dangeardii Dawson, 1954

Chondria sp.

Chondrophycus papillosa (C. Agardh) Garbary and Harper, 1998

Digenea simplex (Wulfen) C. Agardh, 1822

Echinophycus minutus Huisman, 2001

Herposiphonia secunda (C. Agardh) Ambronn, 1880

Laurencia majuscula (Harv.) Lucas 1935

Leveillea jungermannioides (Hering and G. Martens)

Harv., 1855

Osmundaria melvillii (J. Agardh) R.E. Norris, 1991

Polysiphonia ferulacea Suhr ex J. Agardh, 1863

Spirodadia barodensis Børgesen, 1933

Tolypiocladia calodictyon (Harv. ex Kützing) Silva, 1952

Tolypiocladia glomerulata (C. Agardh) F. Schmitz, 1897
Site Number*

37,43

11

59,66

46

38

66

56

37

$11,25,62$

$06,11,17,23,28$

$06,19,23,31,45$

$06,11,45,51,62,68$

62

39,40

69

59

11,31

47

66

$07,11,17,19,23,31,45,51$

62

$05,17,21,48,70$

44

68

03,48

$07,14,41,45,47,66$

DA2/99/32

37

62

$03,23,33,44$

48

48

65

06,11

11,61

\section{RHODYMENIALES}

Champiaceae

Champia compressa Harv., 1838

Champia parvula (C. Agardh) Harveye, 1853

Champia stipitata Huisman, 2000

Champiasp.

\section{Lomentariaceae}

Lomentaria corallicola Børgesen, 1939 
Ceratodictyon spongiosum Zanardini, 1878

Chamaebotrys boergesenii (Weber-van Bosse) Huisman, 1996

Coelarthrum cliftonii (Harv.) Kylin, 1931

Coelarthrum opuntia (End1.) Børgesen, 1937

Coelothrix irregularis (Harv.) Børgesen, 1928

Gelidiopsis intricata (C. Agardh) Vickers, 1905

\section{GIGARTINALES}

Dumontiaceae

Dudresnaya hawaiiensis R.K.S. Lee, 1963

Gibsmithia hawaiiensis Doty, 1963

\section{Hypneaceae}

Hypnea cornuta (Kützing) J. Agardh, 1851

Hypnea pannosa J. Agardh, 1847

Hypnea boergesenii Tanaka, 1941

\section{Nemastomataceae}

Predaea weldii Kraft and Abbott, 1971

\section{Peyssonneliaceae}

Peyssonnelia capensis Montagne, 1847

\section{Rhizophyllidaceae}

Portieria hornemannii (Lyngbye) P.C. Silva, 1987

\section{Schizymeniaceae}

Platoma cyclocolpum (Montagne) F. Schmitz, 1894

Titanophora weberae Børgesen, 1943

\section{Sebdeniaceae}

Sebdenia flabellata (J. Agardh) Parkinson, 1980

\section{Solieriaceae}

Betaphycus speciosum (Sond.) Doty, 1996

Eucheuma denticulatum (N.L. Burman) Collins and

Hervey, 1917

\section{CYANOPHYTA}

Mastigocladaceae

Brachytrichia quoyii (C. Agardh) Bornet and Flahault, 1886

51

\section{Phormidiaceae}

Symploca hydnoides (Harvey) Kütz., 1849

\section{Oscillatoriaceae}

Blennothrix lyngbyacea (Kütz.) Anagnostidis and Komarek, 1988

Lyngbya confervoides C. Agardh, 1824

Lyngbya semiplena (C. Agardh) J. Agardh, 1842
20,25

56

DA2/99/30

DA2/99/29

14,21

20,29

50

27,65

19

$01,10,29$

48

$22,23,46$

$39,40,43,44,46$

$03,05,06,08,09,13,16,18,22,25,27,34,40$, $41,42,43,46,53,58,67$

50

$39,40,46,56,65,69,76$

$47,48,52,65,76$

48

10,48 


\section{MAGNOLIOPHYTA}

\section{Hydrocharitaceae}

Enhalus acoroides (L.f.) Royle, 1839

Thalassia hemprichii (Ehrenb.) Aschers., 1871

Halophila decipiens Ostenfeld, 1902

Halophila minor (Zollinger) Hartog, 1957

Halophila ovalis (R.Br.) J.D. Hooker, 1858
14

$03,10,11,31,45,48,51,62,66$

42, DA2/99/38, DA2/99/66, DA2/99/67, $\mathrm{DA} 2 / 99 / 95-97$

54,62

$37,41,44,45,51,53,58,64$

\section{Potamogetonaceae}

Halodule uninervis (Forssk.) Aschers., 1882

$07,11,31,45,51,62$

\section{REFERENCES}

Huisman, J.M. (1996). The red algal genus Coelarthrum Børgesen (Rhodymeniaceae, Rhodymeniales) in Australian seas, including the description of Chamaebotrys gen. nov. Phycologia 35: 95-112.

Huisman, J.M. (2000). Marine Plants of Australia. University of Western Australia Press, Nedlands.

Huisman, J.M. (2001). Echinophycus minutus (Rhodomelaceae, Ceramiales), a new red algal genus and species from northwestern Australia. Phycological Research 49: 177-182.

Huisman, J.M. (2002). The type and Australian species of the red algal genera Liagora and Ganonema (Liagoraceae, Nemaliales). Australian Systematic Botany 15: 773-838.

Huisman, J.M. and Borowitzka, M.A. (2003). Marine benthic flora of the Dampier Archipelago, Western Australia. In Wells, F.E., Walker, D.I. and Jones, D.S. (eds), Proceedings of the Eleventh International Marine Biological Workshop: The Marine Flora and Fauna of Dampier, Western Australia 2: 291 344. Western Australian Museum, Perth.

Huisman, J.M., Cowan, R.A. and Entwisle, T.J. (1998). Biodiversity of Australian marine macroalgae - a progress report. Botanica Marina 41: 89-93.

King, R.J. and Puttock, C.F. (1994). Macroalgae associated with mangroves in Australia: Rhodophyta. Botanica Marina 37: 181-191.
Lewis, J.A. (1984). Checklist and Bibliography of Benthic Marine Macroalgae recorded from Northern Australia. 1. Rhodophyta. Department of Defence Materials Research Laboratories, Melbourne.

Lewis, J.A. (1985). Checklist and Bibliography of Benthic Marine Macroalgae recorded from Northern Australia. II. Phaeophyta. Department of Defence Materials Research Laboratories, Melbourne.

Lewis, J.A. (1987). Checklist and Bibliography of Benthic Marine Macroalgae recorded from Northern Australia. III. Chlorophyta. Department of Defence Materials Research Laboratories, Melbourne.

Phillips, J.A. (2000). Systematics of the Australian species of Dictyopteris (Dictyotales, Phaeophyceae). Australian Systematic Botany 13: 283-324.

Phillips, J.A. and Huisman, J.M. (1997). Dictyopteris serrata (Dictyotales, Phaeophyceae): a poorly known algal species newly recorded from Australia. Botanica Marina 41: 43-49.

Phillips, J.A., King, R.J., Tanaka, J. and Mostaert, A. (1993). Stoechospermum (Dictyotales, Phaeophyceae): a poorly known algal genus newly recorded in Australia. Phycologia 5: 395-398.

Silva, P.C., Basson, P. and Moe, R. (1996). Catalogue of benthic marine algae of the Indian Ocean. University of California Publications in Botany 79: 1-1279. 